\title{
Interação universidade-empresa e capacidade de aborção em firmas de grande porte do setor de software de Santa Catarina*
}

\author{
Luciana Santos Costa Vieira da Silva \\ Silvio Antonio Ferraz Cario C $^{\ddagger}$ \\ Janaína Ruffoni ${ }^{\S}$
}

\begin{abstract}
Resumo
O presente estudo tem como objetivo avaliar a interação universidade-empresa para desenvolvimento inovativo no setor de software em Santa Catarina. Utiliza-se a abordagem sobre capacidade de absorção de conhecimento externo, como tratamento teórico. Seleciona-se como estudo de caso, cinco grandes empresas atuantes neste setor, localizadas em aglomerações produtivas de Florianópolis, Joinville e Blumenau. A metodologia empregada apresenta as seguintes características: abordagem qualitativa, seleção da amostra intencional e por acessibilidade e utilização de dados de fontes primária e secundária. Os resultados apontam que empresas adotam ações estratégicas voltadas à obtenção de conhecimento externo junto às universidades. As pesquisas desenvolvidas em parceria com universidades resultam em aperfeiçoamento dos produtos existentes, e na introdução de produtos novos pelas empresas no mercado. E, universidades, presentes nas localidades em estudo, constituem fonte para elevar o nível de qualificação dos trabalhadores, cujos conhecimentos adquiridos auxiliam na construção da competência empresarial.
\end{abstract}

Palavras-chave: Interação Universidade-Empresa, Capacidade de Absorção da Firma, Grandes empresas do setor de Software de Santa Catarina.

JEL: 032

\footnotetext{
*Agradecemos as intituições envolvidas.

${ }^{\dagger}$ Professora do Curso de Administração do Centro Universitário Municipal de São José-SC (USJ). Email: luvcosta@gmail.com

${ }^{\ddagger}$ Professor do Depto de Economia e Relações Internacionais e dos Programas de Pós-Graduação em Economia e Administração da Universidade Federal de Santa Catarina (UFSC). Email: fecario@ yahoo.com.br $\S$ Professora do Programa de Pós-Graduação em Economia da Universidade do Vale do Rio dos Sinos (UNISINOS) - Porto Alegre (RS). Email: jruffoni@unisinos.br
} 
62 Capacidade de absorção de conhecimento e a interação universidade-empresa no setor de software do estado de Santa Catarina

\section{Introdução}

O conhecimento constitui um dos principais insumos para o desenvolvimento inovativo e requer condições para ser obtido e transformado no propósito. Condições estas que não são somente internas às empresas, mas dependem da capacidade que se tem de adquiri-lo externamente. Um importante espaço externo para as empresas adquirirem, assimilarem, transformarem e explorarem o conhecimento é a universidade.

No desenvolvimento das atividades econômicas, há segmentos que dependem mais de conhecimento externo. Há os segmentos econômicos que são baseados em ciência, cujas atividades encontram-se constantemente alimentadas por novos saberes, aprendizagens e ideias. Neste grupo se encontram, por exemplo, os segmentos eletrônico, química e software.

Em Santa Catarina, o setor de software encontra-se em franco desenvolvimento e figura entre os principais segmentos de alta tecnologia. Encontra-se fortemente concentrado em três localidades: Blumenau, Joinville e Grande Florianópolis. Esta última possui a maior concentração em número de empresas (187) quando comparada a Blumenau (106) e Joinville (80). Em relação às pessoas empregadas, Blumenau e Joinville contam com maior número atuando em software sob encomenda (1.462 e 1.783, respectivamente) e não-customizável (900 e 424, respectivamente) do que Florianópolis (1.207, sob encomenda; e 793, não-customízável). Ao redor destas empresas existe uma estrutura de ensino, pesquisa e extensão universitária que contribui para o desenvolvimento de suas atividades.

Considerando que o setor de software é classificado entre os que baseiam seu desenvolvimento a partir da ciência, pretende-se analisar neste trabalho, se e como as empresas do setor de software de Santa Catarina recorrem ao conhecimento externo proveniente das universidades e como caracterizam-se as suas capacidades de absorção.

\section{Interação universidade-empresa e capacidade de absorção}

A universidade figura como espaço onde se cria, desenvolve e transfere conhecimento para a sociedade. Para Nelson e Winter (2005, p. 124) esta é “(...) reconhecida como o repositório dos conhecimentos científicos e tecnológicos públicos, conhecimentos estes que elas criam e reproduzem através do ensino e vão aumentando por meio da pesquisa". Também como observa Lee (2000), a universidade colabora com as empresas de diferentes formas com destaque para resolver problemas técnicos, desenvolver novos produtos e processos, melhorar a qualidade dos produtos existentes, reorientar a agenda de P\&D, realizar pesquisa em direção à novas patentes, ter acesso às novas pesquisas através de seminários e workshops; recrutamento de recursos humanos qualificados, entre outros. Segatto-Mendes e Sbragia (2002) apontam que arranjos interativos melhoram a

Econômica-Niterói, v. 20, n. 1, p. 61-76. Junho, 2018 
competitividade das empresas, na medida em que as pesquisas realizadas se traduzem em vantagens competitivas, postas em aumento dos rendimentos e menores custos e riscos empresariais.

Lemos (2013) sintetiza os resultados dos trabalhos de Meyer-Krahmer e Schmoch (1998), Cohen, Nelson e Walsh (2002), Perkmann e Walsh (2007), demonstrando as várias dimensões da interação universidade-empresa. $\mathrm{O}$ primeiro aponta que interações ocorrem a partir de pesquisa colaborativa, contatos informais, formação de pessoal, elaboração de teses, contrato de pesquisa, conferências, consultoria, seminários, intercâmbio de cientistas, publicações e comitês. O segundo registra que as interações ocorrem através de publicações, reuniões, conferências, contratos de pesquisa, consultorias, empreendimentos, patentes, licenças e contratações de graduados. E, o terceiro, destaca links através de parceria de pesquisa, execução de serviços, empreendedorismo, transferência de recursos humanos, interação informal, comercialização de direitos de propriedade e publicação.

Existem trabalhos que procuram apresentar os benefícios que as empresas obtêm a partir da interação com o meio acadêmico universitário. Betts e Santoro (2011) destacam a capacidade de aprender no propósito de melhorar competência para buscar novas tecnologias. Dutrénit e Arza (2010) apontam a implementação de estratégias de inovação de longo prazo, a partir das capacitações construídas. Mueller (2006), Bishop, D’Este e Neely (2011) sinalizam a exploração de novos conhecimentos que alimentam ideias que promovem mudanças técnicas e organizacionais. E, para Meyer-Krahmer e Schmoch (1998), os vínculos elevam a capacidade empresarial de resolução dos problemas técnicos e para absorver pessoas mais qualificadas para seus quadros.

As interações envolvendo empresas e universidade apresentam intensidades diferentes. Malerba e Orsenigo (1993) afirmam que a tecnologia conta com as propriedades de oportunidade tecnológica, cumulatividade do conhecimento, apropriabilidade dos resultados e de conhecimento base. Cassiolato et al. (1996) analisam os vínculos das empresas com universidades, a partir destas propriedades e do padrão tecnológico setorial de Pavitt (1990). Empresas dos setores baseados em ciência - química, eletrônica, software, por ex. - em virtude da dinâmica tecnológica, devem se aproximar do meio acadêmico para atualização e ampliação do conhecimento.

No que diz respeito à capacidade de absorção, essa refere-se à aquisição, assimilação e transformação de conhecimentos para gerar vantagem competitiva (EISENHARDT; MARTIN, 2000; ZAHRA; GEORGE, 2002). Nesta perspectiva, a capacidade de absorção refere-se aos procedimentos para analisar a acumulação e o fluxo de conhecimento em uma empresa (CHANG; HOU; LIN, 2013). O conceito de capacidade de absorção está embutido no debate a respeito das capacidades dinâmicas que foram originadas da teoria evolucionária da empresa (NELSON; WINTER, 2005). Estas últimas permitem às empresas reagirem às mudanças no ambiente de negócios e evitarem a obsolescência das competências (PISANO; TEECE, 2007).

De acordo com Lane, Koka e Pathak (2006), o que Cohen e Levinthal (1989) adici- 
64 Capacidade de absorção de conhecimento e a interação universidade-empresa no setor de software do estado de Santa Catarina

onam à literatura emergente foi um rico conjunto de explicações baseado na economia da organização industrial, de como e porque o P\&D da empresa tem um papel secundário, explicações apoiadas em testes empíricos e um nome conciso e evocativo para aquele papel, ao qual chamaram de capacidade de absorção. Através das atividades de P\&D, a empresa desenvolve conhecimento organizacional sobre certas áreas da ciência e tecnologia, e como essas se relacionam aos produtos e mercados (capacidade de identificar e valorizar o conhecimento, Cohen e Levin (1989)).

Em contribuição a essa discussão, Zahra e George (2002) definem a capacidade de absorção em duas dimensões: a potencial e a realizada. A primeira capta a aquisição e assimilação de conhecimento, condição que permite à empresa identificar e adquirir conhecimento gerado externamente. Enquanto que a segunda, refere-se à capacidade de transformar e explorar os conhecimentos adquiridos para fins comerciais.

A capacidade de absorção potencial reflete a aquisição e assimilação, enquanto que a capacidade de absorção realizada incorpora a noção de Kim (1998) sobre a capacidade de transformação da empresa. Mowery, Oxley e Silverman (1996) afirmam que muitos pesquisadores veem a capacidade de absorção sob duas perspectivas gerais: como estoque de conhecimentos relacionados previamente e na competência de absorver o conhecimento externo. A primeira apresenta-se como estoque, dado que leva em consideração o nível de conhecimento prévio relevante. Em verdade, trata-se da base de conhecimento da empresa. Enquanto a segunda, apresenta-se como expressão da competência que a empresa tem de absorver o conhecimento que está além de seus limites estabelecidos. Segundo Liao et al. (2009), estudos relacionados à capacidade de absorção apresentam elementos centrais relacionados ao: $1^{\mathrm{a}}$.) conhecimento interno incluindo capital humano e tecnologia; $2^{\mathrm{a}}$ ) ambiente externo, tais como políticas e regras governamentais, interações industriais e risco; $3^{\mathrm{a}}$.) gastos em $\mathrm{P} \& \mathrm{D} ; 4^{\mathrm{a}}$.) intensidade de aprendizagem interna; $5^{\mathrm{a}}$.) estratégias organizacionais; e, $6^{\mathrm{a}}$.) processos inovativos como busca de vantagem competitiva.

\section{Aspectos metodológicos}

A pesquisa contempla o estudo de cinco empresas de grande porte do setor de software localizadas em Santa Catarina, mais especificamente em Florianópolis, Joinville e Blumenau. As empresas estudadas são consideradas de grande porte, tomando como referência o faturamento obtido e, na sua maioria, são líderes nos mercados em que atuam. Tais empresas desenvolvem sistemas ERP's (Enterprise Resource Planning).

A seleção da amostra ocorreu de forma intencional e por acessibilidade. A opção por amostras de caráter não probabilístico ou não aleatório justifica-se, principalmente, pela característica qualitativa da pesquisa. $\mathrm{O}$ tratamento dos dados ocorreu por meio de análise documental e de conteúdo.

Econômica-Niterói, v. 20, n. 1, p. 61-76. Junho, 2018 
Tabela 1 - Empresas investigadas do setor de software de Santa Catarina, 2016

\begin{tabular}{llllc}
\hline Empresa & Localização & Porte Empresarial & Cargo do Entrevistado & $\begin{array}{c}\text { Data da } \\
\text { Entrevista }\end{array}$ \\
\hline Alfa & Florianópolis & Grande Empresa & Diretor de Marketing & $25 / 08 / 2016$ \\
Gama & Florianópolis & Grande Empresa & Assessor de Projetos Especiais & $16 / 09 / 2016$ \\
Ômega & Blumenau & Grande Empresa & Coordenador de Inovação & $05 / 10 / 2016$ \\
Delta & Joinville & Grande Empresa & Diretor de Projetos & $31 / 10 / 2016$ \\
Lambda & Blumenau & Grande Empresa & $\begin{array}{l}\text { Diretor de Desenvolvimento } \\
\text { de software }\end{array}$ & $07 / 11 / 2016$ \\
& & & & \\
\hline
\end{tabular}

Fonte: Elaborado pelos autores

\section{Interação universidade-empresa e capacidade de absorção}

\subsection{Características das empresas investigadas, suas interações e capacidade de ab- sorção}

A empresa Alfa, criada em 1990, encontra-se localizada em Florianópolis. Possui cerca de 2.800 clientes, conta com mais de 1.500 colaboradores e tem um faturamento médio anual de R $\$ 70$ milhões. É especializada no desenvolvimento e na implantação de softwares de gestão para os segmentos de justiça, infraestrutura, gestão pública e construção civil. Dentre as inovações geradas, destaca-se uma patente registrada de software para construção civil.

A empresa Gama, também localizada em Florianópolis, iniciou suas atividades fornecendo produtos para telecomunicações. Seu portfólio de produtos atual inclui software e hardware de Inteligência Corporativa e Investigativa, Call Center e Contact Center, Convergência de Redes e PABX, além de serviços em tecnologia de informação e telecomunicações. Comercializa plataformas de Computer \& Telephone Integrated (CTI) e para redes corporativas e órgãos de segurança pública. O faturamento anual da empresa é de cerca de R \$ 100 milhões e conta com 684 funcionários, sendo que 75\% estão alocados na atividade fim da empresa. Realiza parcerias com instituições de ensino superior, sendo destaque as interações com a Universidade Federal de Santa Catarina (UFSC) e a Universidade Federal do Ceará (UFCE). Possui dois registros de patentes: analisador digital de sinais multifrequências e equipamento para mala direta.

A empresa Delta, situada em Blumenau, auta no setor de software há 29 anos. O curso de graduação em ciências da computação da Fundação Universidade Regional de Blumenau (FURB) e a demanda crescente de empresas locais pelos seus produtos auxiliam no desenvolvimento da empresa. Trabalha com softwares de gestão empresarial nos segmentos de softwares de gestão de pessoas, acesso a segurança e de logística, bem como desenvolve softwares de performance corporativa, os "BIs", workflows e Dashboard. A 
empresa realiza parcerias com institutos de pesquisa como a Fundação Centro de Referência em Tecnologias Inovadoras (CERTI) e Universidades, dentre as quais a Universidade do Sul de Santa Catarina (UNISUL), a UFSC e a FURB, no propósito de desenvolver $\mathrm{P} \& \mathrm{D}$ no modelo de inovação aberta. A empresa conta com cerca de 10 mil clientes e o faturamento anual, do grupo que pertence, situa-se em torno de $\mathrm{R} \$ 56$ milhões.

A empresa Ômega é fruto da fusão de uma empresa localizada em São Paulo (SP) e a outra em Joinville (SC). Em 2011, criou a Ômega Labs no centro de pesquisa de soluções em computação em nuvem, "cloud computing", no Campus da Universidade da Califórnia. Entre os anos de 2013 e 2014 adquiriu oito empresas de software no Brasil e uma outra nos EUA. A área de atuação volta-se para o desenvolvimento e comercialização do direito de uso de aplicativos para gestão empresarial, Enterprise Resource Planning (ERP). Além disso, comercializa serviços de adaptação dos softwares a pedido de cliente e realiza implantação, treinamento, consultoria e manutenção de sistemas. Possui elevada participação no mercado de software de gestão na América Latina, 34,5\%, e detém a liderança no Brasil, com 48,6 \% de fatia do mercado. O faturamento anual gira ao redor de R \$ 1,7 bilhão. A empresa Ômega possui 9.000 funcionários, sendo 1.100 em Joinville, dos quais 200 dedicados à área de inovação. Conta com patente registrada no Instituto Nacional de Propriedade Industrial (INPI) desde 2007, referente à métodos e sistemas para ciclo de vendas e de implementação de software.

A empresa Lambda, iniciou suas atividades e 1997 e localiza-se em Blumenau. Atualmente oferece soluções em software, serviços e gestão de processos administrativos. Também cria soluções nas áreas de logística e supply chain, turismo e de saúde - operadoras e hospitalar - com foco em tecnologia, gestão de sinistro, terceirização e serviço de orientação médica (nurse call). Conta com cerca de 1.200 colaboradores, figura entre as cinco maiores players no mercado de software de gestão empresarial no Brasil. Em 2015, o grupo que pertence a empresa Lambda registrou faturamento da ordem de R \$ 195 milhões.

A empresa Alfa considera relevante as interações entre os atores pertencentes ao sistema de inovação. Os vínculos existentes com empresas privadas, institutos de pesquisa e/ou universidades criam condições para o estabelecimento de projetos conjuntos, troca de informações, transferência de conhecimento, contratação de pessoal qualificado, entre outros aspectos que possibilitam desenvolver inovações. A afirmação de um entrevistado destaca: "Nós temos parcerias de fornecimento de tecnologia com os grandes players mundiais, Oracle, IBM, também com universidades, principalmente com a UFSC que é o principal parceiro".

As parcerias que a empresa Alfa realiza com universidades são mais intensas na formação de mão-de-obra qualificada para atuar na empresa. A UFSC é, em grande monta, responsável pela formação qualificada de pessoas atuantes na área de software da região da Grande Florianópolis. No entanto, também são realizadas parcerias na área de pesquisa: “(...) então hoje a maior parte das parcerias que nós temos com as Instituições de 
Ensino é para a formação de mão de obra qualificada pra atuar na empresa, mas também tem algumas parcerias tecnológicas com a UFSC."

Uma adaptabilidade proativa da empresa ocorre ainda na década de 1990 quando o conceito de "Cloud Computing" ainda não era uma realidade no Brasil. Com o propósito de desenvolver pesquisa nesta área, estabeleceu parceria com a UFSC, voltada em proporcionar maior segurança aos dados, possibilitando, assim, seus clientes postarem conteúdo na nuvem. Essa aposta deu à empresa o pioneirismo no mercado de ERP em nuvem, conforme declarou o entrevistado: “(...) nós temos 1400 empresas operando em nuvem, então, em termos de Brasil, não tem outra empresa assim e, em termos mundiais, é um case bastante significativo". Em 2007, o Instituto Stela, laboratório do Programa de PósGraduação em Engenharia de Produção (PGEP) da UFSC, requisita à empresa auxílio para a manutenção da plataforma que integra dados da Coordenação de Aperfeiçoamento de Pessoal de Nível Superior (CAPES), do Conselho Nacional de Desenvolvimento Científico e Tecnológico (CNPq) e da Universidade com conexão Web. Esta parceria constitui uma das primeiras experiências de inovação aberta do Instituto Stela. Em 2011, outra expressão desta parceria se manifesta pelo uso dos produtos da empresa. A UFSC tornase cliente do software de gestão pública da empresa Alfa, cujo produto teve participação de pessoas formadas por esta instituição de ensino superior. A interação permite que ocorra participação mútua de pesquisadores da empresa e da universidade, na geração do produto. Afirmações do representante da empresa Alfa apontam o reconhecimento da melhoria das condições competitivas, a partir dos relacionamentos firmados com a instituição de ensino superior.

Na empresa Gama, a interação realizada com universidades, ocorre não só com o propósito de contratar profissionais formados e em formação dos cursos de ciências da computação, sistemas de informação, engenharia e outros da UFSC, mas também em parcerias realizadas com diversos laboratórios desta universidade para desenvolver pesquisa tecnológica.

Segundo o entrevistado, a universidade realiza pesquisa científica e a empresa oferece infraestrutura interna, para que o conhecimento gerado possa ser colocado em prática. A empresa possui vínculos com a Fundação Centros de Referência em Tecnologias Inovadoras (CERTI), o Laboratório de Integração de Software e Hardware (LISHA) e o Laboratório de Circuitos e Processamento de Sinais (LINSE). A interação com o laboratório LINSE da UFSC, constitui a maior referência de vínculo para a empresa entre o meio acadêmico e o meio empresarial.

"A empresa trabalha desde 90 com o laboratório LINSE (Laboratório de Circuitos e Processamento de Sinais) da universidade (UFSC). Ela trabalha com outros CERTI, LISHA (Laboratório de Integração de Software e Hardware), etc, etc, mas o LINSE ela já tem uma relação muito longa, pra nós é o melhor laboratório que tem lá, que trabalha neste segmento".

Econômica-Niterói, v. 20, n. 1, p. 61-76. Junho, 2018 
Outras parcerias com universidades são realizadas nos últimos anos, como as firmadas com a Universidade Federal do Ceará (UFCE) e o Serviço Nacional da Indústria (SENAI). Tais parcerias expressas no propósito de realização de pesquisa e de qualificação de trabalhadores, segundo entrevistado, visam criar melhores condições para o desenvolvimento inovativo interno. No setor de $\mathrm{P} \& \mathrm{D}$, os colaboradores são incentivados à realizar pesquisas em bases de dados de periódicos internacionais, a fim de identificar a tecnologia que está sendo cientificamente estudada no momento. Nesta rotina, os pesquisadores recorrem a bases de dados como Elsevier, Scopus, Science Direct e Thompson Reuters, no propósito de auxiliar na busca de soluções para os problemas tecnológicos da empresa. Neste sentido, o entrevistado relata:

\footnotetext{
“(...) vê-se qual é a tecnologia, faz-se o gap de análise e capacita a turma que vai fazer pelo que se achou. Então a turma de P\&D tem que saber pesquisar. A turma que desenvolve a inovação tem que saber a tecnologia que essa turma que pesquisou descobriu".
}

A qualificação dos colaboradores ocorre através de incentivos para realizar cursos de graduação e pós-graduação em área de conhecimento vinculada ao core competence da empresa. Para a qualificação relacionada à realização de cursos no stricto sensu, como mestrado e doutorado, o incentivo da empresa se vincula a ceder o colaborador nos dias em que ele possui atividades acadêmicas.

A empresa Delta segue orientação interna de se relacionar com universidades através da formação de mão-de-obra dos colaboradores. Para tanto, disponibiliza regime de incentivo por meio de concessão de bolsas de estudo e de isenção de horas de trabalho. $O$ entrevistado relata como ocorre a interação da empresa com as Universidades:

\footnotetext{
"O acesso [...] ao conhecimento com [...] a universidade [...] vem muito como formação de mão-de-obra de funcionário. Então assim, [...] tem um programa que apoia o funcionário com bolsas de até $50 \%$ para que eles possam estudar em universidades privadas. Em universidades públicas também tem o apoio, mas é com a isenção de horas de trabalho, se ele precisar $[\ldots] "$.
}

A exploração de linhas de incentivo também se estende através de obtenção de recursos do CNPq, no programa de Formação de Recursos Humanos em Áreas Estratégicas (RHAE). A afirmação do entrevistado desta empresa referenda esta observação: "No passado a gente teve uma experiência com o CNPq utilizando o pesquisador RHAE".

Além desta fonte de financiamento, a empresa recorre ao regime de incentivo da Lei do Bem e das linhas de financiamento do Banco Nacional de Desenvolvimento Econômico e Social (BNDES) para P\&D, como forma de desenvolver o potencial tecnológico interno. Neste contexto, buscam-se recursos com o intuito de melhorar as condições física, técnica e pessoal da infraestrutura laboratorial: "O maior benefício, digamos assim, no P\&D 
da empresa vem da Lei do Bem e das linhas de financiamento do BNDES". Com tal empreendimento, criam-se condições para maior aproximação dos meios empresarial e acadêmico em trocas de informação, geração de conhecimento e formas de aprendizado. A empresa também focaliza suas estratégias na universidade corporativa, como forma de integrar as pessoas em diferentes setores. A universidade corporativa disponibiliza plataforma de ensino à distância aos usuários, conforme ressalta o entrevistado:

\begin{abstract}
“(...) a gente tem uma estrutura aí com a plataforma EAD muito bem estruturada, aonde todas as tecnologias e soluções e tudo que é de conhecimento novo, tanto na parte tecnológica e na parte de negócio, tanto para a parte, para a equipe técnica como para a equipe comercial, é gerenciada, é gerida pela universidade corporativa)".
\end{abstract}

Para a empresa Delta, os elementos citados condizem com a formação de capacidades específicas aos membros organizacionais, que podem se tornar dinâmicas e gerarem novos produtos e serviços oferecidos. Contribui de forma decisiva para tal ocorrência, o fato da empresa estar inserida num sistema de inovação, em que além da interação com universidades dentre as quais a UFSC e UFCE, conta com outras instituições importantes de apoio, como as de financiamento - CNPq e BNDES - e regulamentação incentivadora, como a Lei do Bem.

Dentre as interações estabelecidas pela Ômega no âmbito de um sistema de inovação, a empresa dá preferência às relações que se firmam com o mercado. O comportamento em atuar orientada ao cliente consiste na criação de segmentos específicos para atender nichos diferentes de empresas, como a área de manufatura, logística, jurídico, agroindustrial, entre outros. No Brasil, a empresa Ômega considera a universidade apenas como instância fornecedora de conhecimento para trabalhadores, e não como espaço que possibilita vínculos interativos voltados ao desenvolvimento da pesquisa. Neste sentido, o entrevistado afirma:

\footnotetext{
"Eu vejo que a ÔMEGA não tem tanto histórico de fazer parcerias [...]com universidades. [...] tem muita gente que procura a ÔMEGA pra fazer parceria porque a gente tem muitos clientes [...], e isso abre um leque muito grande pra essas empresas... tem várias empresas que buscam muito isso...então eu acho que é por isso que a ÔMEGA não procura tantas parcerias, [...]...as parcerias é que vêm até ela".
}

Tal posicionamento decorre de a empresa apresentar um volume significativo de clientes e valer-se, para tanto, mais da relação produtor de software e usuário, do que interações com universidades e institutos de pesquisa, para desenvolvimento tecnológico de seus produtos e serviços. A trajetória de desenvolvimento percorrida contribui para este posicionamento, pois são décadas atuando no segmento de software. Mas essa relação começou a mudar a partir 2010, quando a empresa estabeleceu parceria com duas universidades na Califórnia (EUA): A Universidade de Stanford e San Jose State (SJSU). A 
70 Capacidade de absorção de conhecimento e a interação universidade-empresa no setor de software do estado de Santa Catarina

parceria com a Universidade de Stanford tem o objetivo de criar novos negócios no mercado americano, enquanto que o vínculo com a Universidade San Jose State possibilita realizar estudos a respeito do ambiente de desenvolvimento dos softwares.

A empresa Lambda já realizou no passado interação com as universidades. Naquela oportunidade, vínculos ocorreram para a realização de pesquisas de uso imediato. Uma vez atendida a demanda, encerrou-se o contrato firmado. A afirmação do entrevistado sinaliza neste sentido: "[...] a gente já teve algumas experiências, mas não temos nada efetivamente. [...]". No momento, vínculos com o meio acadêmico através de projetos de pesquisa não ocorrem.

Por sua vez, as relações estabelecidas pela empresa com a universidade estão vinculadas na área do ensino, por meio da frequência de seus trabalhadores nos cursos de graduação de uma universidade local:

\footnotetext{
“(...) a gente teve experiências no sentido de fornecer o nosso produto para que usassem no curso de administração; por exemplo, usando o produto da Lambda, isso a gente fez aqui na FURB, tínhamos até recentemente uma negociação acontecendo com o Instituto Catarinense de Educação aqui em Blumenau, pra usar os produtos da Lambda como parte da aula, geralmente no curso de administração".
}

Além disso, uma das formas encontradas para buscar informações pela empresa ocorre por meio de consultorias. Estas são contratadas para obtenção de informações sobre as tendências do mercado que atendem às necessidades dos clientes. Assim como, realiza parcerias técnicas com empresas privadas, para complementar os produtos e/ou serviços oferecidos. Através destes mecanismos, as empresas trocam informações tecnológicas importantes visando alcançar objetivos comuns.

\subsection{Análise das Interações Estabelecidas e Capacidades de Absorção das Firmas}

As empresas em estudo recorrem às universidades para terem apoio no desenvolvimento de suas atividades. Um ponto comum em todas as empresas investigadas refere-se à contratação de profissionais formados nas áreas de conhecimento da computação, engenharia, administração e sistema de informação pelas universidades, situadas, mas não só, no local e região em que se encontram.

As empresas - Alfa, Gama, Delta, Ômega e Lambda -, identificam e valorizam, de forma explícita, o conhecimento externo proveniente das universidades. Um dos procedimentos utilizados refere-se em criar condições para que seus trabalhadores obtenham titulação universitária. Também, firmam convênios com universidades para seus trabalhadores realizarem cursos de pós-graduação, em área de interesse empresarial.

Da mesma forma, as empresas - Alfa, Gama, Delta e Ômega - interagem com as universidades para desenvolvimento de pesquisa orientadas em solucionar problemas, aperfeiçoar sistemas, auxiliar na criação de novos produtos entre outros propósitos. Para além

Econômica-Niterói, v. 20, n. 1, p. 61-76. Junho, 2018 
das capacidades de assimilação e absorção, conforme Zahra e George (2002), tais empresas demonstram as capacidades de transformação e exploração do conhecimento externo, recorrendo a parcerias com o meio acadêmico. Papel importante neste processo, são os vínculos formados pelas empresas com os laboratórios, departamentos e fundações da UFSC. Citam-se os arranjos efetuados pela empresa Gama com os laboratórios LISHA e LINSE e a fundação CERTI e os vínculos estabelecidos da empresa Alfa com o Instituto Stela, constituem referências.

Contribui de forma efetiva no processo interativo das empresas com as universidades, o sistema de inovação nacional. Há registros do CNPq e da CAPES concederem recursos para pagamento de custos operacionais de pesquisa no propósito de incentivar os esforços inovativos. Assim como, apontamentos do BNDES e da Lei do Bem auxiliando as empresas na montagem e modernização de laboratórios de $\mathrm{P} \& \mathrm{D}$. Tais ocorrências seguem as observações de Edquist (2001), que os integrantes de um sistema de inovação agem no propósito de criar condições para desenvolvimento e difusão do conhecimentos próinovação. Da mesma forma, tais ações institucionais estão em linha com os escritos de Bosch, Volberda e Boer (1999), de que as empresas identificam, assimilam e exploram o conhecimento considerando as economias de custos e de escala que podem obter.

No desenvolvimento das interações das empresas de software e as universidades devese considerar, conforme Zahra e George (2002), Xia e Roper (2008), os resultados alcançados. As empresas consideram no âmago dos processos de assimilação, absorção, transformação e exploração do conhecimento, a taxa e a efetividade dos processos resultantes. Os resultados expressam linhas do progresso técnico obtidas a partir de esforços mútuos realizados. Constroem-se path-dependencies relacional e linhas de processos de aprendizagem. Prova disto é a temporalidade das interações: as empresas Alfa e a Gama realizam parcerias com universidades desde a década de 1990. A Delta mantém parcerias a partir da década de 2000. A Lambda sustenta a interação desde 2009 e a Ômega realiza vínculos pós o ano 2010.

Os produtos inovadores gerados a partir das interações dos meios empresarial e o acadêmico, comprovam a virtuosidade das relações firmadas. Na empresa Alfa, a parceria com a UFSC resultou no desenvolvimento de tecnologia para segurança de dados em nuvem. Na empresa Gama, a interação possibilitou a criação do taquígrafo que transcreve automaticamente áudio. Na empresa Delta, vínculos firmados possibilitam seus pesquisadores obterem os títulos de mestre e doutor, bem como, resultam em auxílio no desenvolvimento do projeto de universidade corporativa. Na empresa Ômega, as parcerias com Stanford e San Jose State, possibilitam o desenvolvimento de ferramentas e metodologias para criação de novos softwares. E, na empresa Lambda, a interação com a FURB permitem o uso de seus produtos nos processos de aprendizagem dos estudantes universitários.

Os resultados inovativos apresentados devem ser considerados à luz dos procedimentos adotados pelas empresas. Em linha com os escritos de: Todorova e Durisin (2007), as 
empresas em estudo, reconhecem e dão importância ao conhecimento externo, realizando vínculos interativos com suas fontes geradoras. Bem como, criam condições domésticas para assimilar o conhecimento externo e inter-relacioná-lo com o interno, em processos de mudança das estruturas cognitivas existentes. Na mesma perspectiva, a quase totalidade das empresas pesquisadas empresas posiciona favoravelmente aos escritos de Liao et al. (2009): primeiro, por eleger o processo inovativo como caminho para obtenção de vantagem competitiva (com exceção de apenas uma que privilegia a concorrência via preço e valoriza relativamente menos as interações com universidades do que as demais); e, segundo, por contarem com condições infraestruturais internas, dentre as quais laboratórios, equipamentos, recursos financeiros e pessoal qualificado para absorver o conhecimento externo.

Portanto, observou-se nas interações identificadas que a Universidade exerce tanto sua função tradicional de ensino, quanto suas funções de pesquisa e extensão (no papel de ensino corporativo, por exemplo). Destaca-se também que as empresas investigadas são de grande porte, algo que resulta em viés nos achados da pesquisa, no sentido de valorizarem e usarem fontes externas de conhecimento, algo pouco observado na Pesquisa de Inovação (PINTEC) - do Instituto Brasileiro de Geografia e Estatística (IBGE) - que informa o padrão de inovação das firmas brasileiras. Há certo consenso na literatura que empresas de grande porte, na média, possuem melhores condições para absorver conhecimentos, interagir com outros agentes do sistema de inovação e inovar.

\section{Conclusões}

O presente estudo demonstra que as empresas de grande porte do setor de software investigadas fazem uso da capacidade de absorção de conhecimento externo, para promoverem melhores condições internas de desenvolvimento de suas atividades. Possuem ações voltadas para a busca deste tipo de conhecimento no ambiente institucional que as cerca. A presença de integrantes do sistema de inovações - universidades, governo, instituições de fomento e sistema financeiro - auxilia na concretização deste propósito.

Sintetizam-se os principais resultados do estudo nos seguintes pontos: (a) vínculos interativos de longo prazo, com registro de contratos com algumas empresas há mais de 2 décadas; (b) contribuição virtuosa das universidades e seus laboratórios no desenvolvimento técnico e inovativo de produtos e serviços das empresas; (c) formação de mão-deobra qualificada pelas universidades para atuação nas áreas da tecnologia de informação e de administração das empresas; (d) prestação de serviços pelas empresas nos laboratórios e estruturas administrativas das universidades para aperfeiçoamento de suas atividades; (e) desenvolvimento de mentalidade de esforços cooperativos para busca inovativa pelos meios empresariais e universitários; (f) esforços das empresas na criação de infraestrutura tecnológica - laboratório, pessoal e recursos - para melhor desenvolver a capacidade de absorção; (g) incorporação de novas procedimentos de busca e de rotina em opera-

Econômica-Niterói, v. 20, n. 1, p. 61-76. Junho, 2018 
ções voltadas ao desenvolvimento inovativo pelas empresas; e, (h) reconhecimento da contribuição da universidade na obtenção de registros de patentes de produtos e serviços inovadores pelas empresas.

Demonstra-se, enfim, que o processo de construção do conhecimento de uma empresa deve levar em consideração não somente sua estrutura interna, mas também as fontes externas de conhecimentos que são capazes de absorver. Este condicionante soma-se à consideração que o processo inovativo é sistêmico, envolvendo vários atores.

\begin{abstract}
The presente study aims to evaluate the university-industry interaction for innovative development in the software sector in Santa Catarina. Use the approach on the absorption capacity of external knowledge, as theoretical treatment. Five large companies operating in this sector are selected, as case study, located in productive clusters of Florianópolis, Joinville and Blumenau. The methodology used has the following characteristics: qualitative approach, selections of the intentional and accessibility sample and use of data from primary and secondary source. The results indicate that firms adopt strategic actions aimed at obtaining external knowledge from universities. Researches developed in partnership with universities result in the improvement of existing products, and the introduction of new products by firms in the market. And, universities, present in the cluster studied, constitute a source to raise the level of formal qualification of the workers, whose acquired knowledge helps in the construction of the entrepreneurial competence.
\end{abstract}

Keywords: Absorptive Capacity, Relation University-Industry, Software Sector from Santa Catarina, development.

JEL: 032

\title{
Referências bibliográficas
}

BETTS, S. C.; SANTORO, M. D. Somewhere between markets and hierarchies: controlling industry university relationships for success. Academy of Strategic Management journal, v. 10, n. 1, 2011.

BISHOP, K.; D'ESTE, P.; NEELY, A. Gaining from interactions with universities: Multiple methods for nurturing absorptive capacity. Research Policy, v. 40, n. 1, p. 30-40, 2011.

BOSCH, F. A. J. V. d.; VOLBERDA, H. W.; BOER, M. d. Coevolution of Firm Absorptive Capacity and Knowledge Environment: Organizational Forms and Combinative Capabilities. Organization Science, v. 10, n. 5, p. 551-568, 1999. Disponível em: <https://www.jstor.org/stable/2640317>. 
$74 \quad$ Capacidade de absorção de conhecimento e a interação universidade-empresa no setor de software do estado de Santa Catarina

CASSIOLATO, J. E. et al. A relação universidade e instituições de pesquisa com o setor industrial: uma análise de seus condicionantes. [S.1.]: IE/UFRJ - Mimeo, 1996.

CHANG, H.-J.; HOU, J.-J.; LIN, S.-J. A multi-cases comparative approach on forming Elements of dynamic capability. International Journal of Organizational Innovation, v. 5, n. 4, p. 52-64, 2013. Disponível em: <http://eds.b.ebscohost.com/eds/detail/detail? vid=0\&sid=ae4fdf3b-dfe5-48b5-9578-25398ec527851\%40sessionmgr120\&bdata $=$ JkF1dGhUeXB1PWNvb2tpZSxpcCxzaGliJmxhbmc9cHQtYnImc210ZT11ZHMtbG12ZQ। $\% 3 \mathrm{~d} \backslash \% 3 \mathrm{~d} \backslash \# \mathrm{AN}=90515713 \& \mathrm{db}=\mathrm{bsu}>$.

COHEN, W. M.; LEVIN, R. C. Empirical studies of innovation and market structure. In: SCHMALENSEE, R.; WILLIG, R. (Ed.). Handbook of Industrial Organization. Elsevier, 1989. v. 2, cap. 18, p. 1059-1107. Disponível em: $<$ https://ideas.repec.org/h/eee/indchp/2-18.html>.

COHEN, W. M.; NELSON, R. R.; WALSH, J. P. Links and Impacts: The Influence of Public Research on Industrial R\&amp;D. Management Science, INFORMS, v. 48, n. 1, p. 1-23, 1 2002. Disponível em: <http://pubsonline.informs.org/doi/abs/10.1287/mnsc. 48.1.1.14273>.

DUTRÉNIT, G.; ARZA, V. Channels and benefits of interactions between public research organisations and industry: comparing four Latin American countries. Science and Public Policy, Oxford University Press, v. 37, n. 7, p. 541-553, 8 2010. Disponível em: <https://academic.oup.com/spp/article-lookup/doi/10.3152/030234210X512043>.

EDQUIST, C. Systems of Innovation for Development (SID). [s.n.], 2001. Disponível

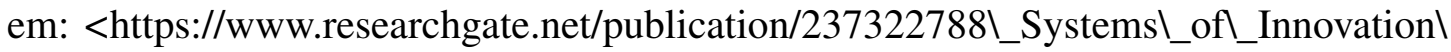

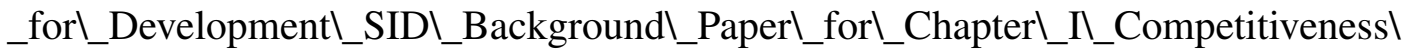

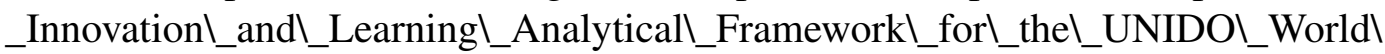
_Industrial\_Development $\ \_R e p o r t l \_W I D R \backslash 2001>$.

EISENHARDT, K. M.; MARTIN, J. A. Dynamic capabilities: what are they? Strategic Management Journal, Wiley-Blackwell, v. 21, n. 10-11, p. 1105-1121, 102000. Disponível em: <http://doi.wiley.com/10.1002/1097-02661\%28200010/111\%2921। \%3A10/11\\%3C1105\\%3Al\%3AAID-SMJ133\\%3E3.0.CO\\%3B2-E>.

KIM, D. H. The Link between Individual and Organizational Learning. The Strategic Management of Intellectual Capital, Butterworth-Heinemann, p. 4162, 1 1998. Disponível em: <https://www.sciencedirect.com/science/article/pii/ B9780750698504500063>.

LANE, P. J.; KOKA, B. R.; PATHAK, S. The Reification of Absorptive Capacity: A Critical Review and Rejuvenation of the Construct. Academy of Management, 2006. 833-863 p. Disponível em: <https://www.jstor.org/stable/20159255>.

Econômica-Niterói, v. 20, n. 1, p. 61-76. Junho, 2018 
LEE, Y. S. The Sustainability of University-Industry Research Collaboration: An Empirical Assessment. The Journal of Technology Transfer, Kluwer Academic Publishers, v. 25, n. 2, p. 111-133, 2000. Disponível em: <http://link.springer.com/10. 1023/A:1007895322042>.

LEMOS, D. d. C. A interação universidade-empresa para o desenvolvimento inovativo sob a perspectiva institucionalista-evolucionária: uma análise a partir do sistema de ensino superior em Santa Catarina. Tese (Doutorado) - UFSC, 2013. Disponível em: $<$ https://repositorio.ufsc.br/handle/123456789/122848>.

LIAO, S.-H. et al. Knowledge Acquisition, Absorptive Capacity, and Innovation Capability: An Empirical Study of Taiwan's Knowledge-Intensive Industries. World Academy of Science, Engineering and Technology, v. 53, p. 160-167, 2009. Disponível em: <http://connection.ebscohost.com/c/articles/41562204/>.

MALERBA, F; ORSENIGO, L. L'accumulazione delle capacita tecnologiche nell industria italiana (1969-1984). In: FILIPPINI, C. (Ed.). Innovazione tecnologica e servizi alle imprese. 1. ed. Milán: Franco Angeli, 1993. ISBN 9788820482787.

MEYER-KRAHMER, F.; SCHMOCH, U. Science-based technologies: university-industry interactions in four fields. Research Policy, v. 27, n. 8, p. 835-851, 12 1998. ISSN 00487333. Disponível em: $<$ http://linkinghub.elsevier.com/retrieve/pii/S0048733398000948>.

MOWERY, D. C.; OXLEY, J. E.; SILVERMAN, B. S. Strategic alliances and interfirm knowledge transfer. Strategic Management Journal, Wiley-Blackwell, v. 17, n. S2, p. 77-91, 12 1996. Disponível em: <http://doi.wiley.com/10.1002/smj.4250171108>.

MUELLER, S. P. M. A comunicação científica e o movimento de acesso livre ao conhecimento. Ciência da Informação, IBICT, v. 35, n. 2, p. 27-38, 8 2006. Disponível em: <http://www.scielo.br/scielo.php?script=scil_arttext\&pid= S0100-19652006000200004\&lng=pt\&tlng=pt>.

NELSON, R. R.; WINTER, S. G. Uma Teoria Evolucionária da Mudança Econômica. Campinas: Unicamp, 2005. 632 p. ISBN 8526807129. Disponível em: <https: //www.saraiva.com.br/uma-teoria-evolucionaria-da-mudanca-economica-194603.html>.

PAVITT, K. What We Know about the Strategic Management of Technology. California Management Review, SAGE PublicationsSage CA: Los Angeles, CA, v. 32, n. 3, p. 17-26, 4 1990. Disponível em: <http://journals.sagepub.com/doi/10.2307/41166614>.

PERKMANN, M.; WALSH, K. University-industry relationships and open innovation: Towards a research agenda. International Journal of Management Reviews, Wiley/Blackwell, v. 9, n. 4, p. 259-280, 12 2007. Disponível em: $<$ http://doi.wiley.com/10.1111/j.1468-2370.2007.00225.x>. 
76 Capacidade de absorção de conhecimento e a interação universidade-empresa no setor de software do estado de Santa Catarina

PISANO, G.; TEECE, D. J. Knowledge integrators and the survival of manufacturing clusters. California Management Review, v. 50, n. 1, p. 1069-1089, 2007. Disponível em: <https://academic.oup.com/joeg/article/18/5/1069/5045539>.

SEGATTO-MENDES, A. P.; SBRAGIA, R. O processo de cooperação universidadeempresa em universidades brasileiras. Revista de Administração, v. 37, n. 4, p. 58-71, 2002. Disponível em: <http://www.contextus.ufc.br/2014/index.php/contextus/article/ view/674>.

TODOROVA, G.; DURISIN, B. Absorptive Capacity: Valuing a Reconceptualization. The Academy of Management Review, Academy of Management, v. 32, n. 3, p. 774-786, 2007. Disponível em: <https://www.jstor.org/stable/20159334>.

XIA, T.; ROPER, S. From capability to connectivity-Absorptive capacity and exploratory alliances in biopharmaceutical firms: A US-Europe comparison. Technovation, Elsevier, v. 28, n. 11, p. 776-785, 11 2008. Disponível em: $<$ http://linkinghub.elsevier.com/retrieve/pii/S0166497208000448>.

ZAHRA, S. A.; GEORGE, G. Absorptive Capacity: A Review, Reconceptualization, and Extension. The Academy of Management Review, Academy of Management, v. 27, n. 2, p. 185, 4 2002. Disponível em: <http://links.jstor.org/sici?sici=0363-7425\\%28200204\ $\% 2927 \backslash \% 3 \mathrm{~A} 2 \backslash \% 3 \mathrm{C} 185 \backslash \% 3 \mathrm{AACARRA} \% 3 \mathrm{E} 2.0 . \mathrm{CO} \backslash \% 3 \mathrm{~B} 2-6 \&$ origin=crossref $>$. 